\title{
Mycophenolic acid, the active form of mycophenolate mofetil, interferes with IRF7 nuclear translocation and type I IFN production by plasmacytoid dendritic cells
}

Minoru Shigesaka, Tomoki Ito* (10, Muneo Inaba, Kai Imai, Hideki Yamanaka, Yoshiko Azuma, Akihiro Tanaka, Hideki Amuro, Tohru Nishizawa, Yonsu Son, Atsushi Satake, Yoshio Ozaki and Shosaku Nomura

\begin{abstract}
Background: Both humoral and cellular immune mechanisms are involved in the onset and progression of autoimmune responses in systemic lupus erythematosus (SLE). Plasmacytoid dendritic cells (pDCs) play a central role in the pathogenesis of SLE via the dysregulation of type I interferon (IFN) production; these cells act together with activated myeloid DCs (mDCs) to amplify the vicious pathogenic spiral of autoimmune disorders. Therefore, control of aberrant DC activation in SLE may provide an alternative treatment strategy against this disease. Mycophenolate mofetil (MMF), which has been used to treat lupus nephritis, specifically blocks the proliferation of B and T lymphocytes via inhibition of inosine-5-monophosphate dehydrogenase. Here, we focus on the effects of MMF in targeting DC functions, especially the IFN response of pDCs.

Methods: We isolated human blood pDCs and mDCs by flow cytometry and examined the effect of mycophenolic acid (MPA), which is a metabolic product of MMF, on the toll-like receptor (TLR) ligand response of DC subsets. Additionally, we cultured pDCs with serum from SLE patients in the presence or absence of MPA and then examined the inhibitory function of MPA on SLE serum-induced IFN-a production.

Results: We found that treatment with 1-10 $\mu \mathrm{M}$ of MPA (covering the clinical trough plasma concentration range) dose-dependently downregulated the expression of CD80 and CD86 on mDCs (but not pDCs) without inducing apoptosis, in response to R848 or CpG-ODN, respectively. Notably, in pDCs, MPA significantly suppressed IFN-a production with IRF7 nuclear translocation and repressed the AKT activity. In addition, MPA inhibited IL-12 production with STAT4 expression in mDCs. We further identified that MPA had an inhibitory effect on SLE seruminduced IFN-a production by pDCs.

Conclusions: Our data suggest that MPA can interrupt the vicious pathogenic spiral of autoimmune disorders by regulating the function of DC subsets. This work unveiled a novel mechanism for the therapeutic ability of MMF against SLE.
\end{abstract}

Keywords: SLE, Plasmacytoid DCs, Myeloid DCs, Type I IFN, MPA, IRF7

\footnotetext{
* Correspondence: itot@hirakata.kmu.ac.jp

First Department of Internal Medicine, Kansai Medical University, 2-5-1

Shin-machi, Hirakata City, Osaka 573-1010, Japan
} 


\section{Background}

Mycophenolate mofetil (MMF), which has been used to treat lupus nephritis as well as in solid organ transplantation, is an uncompetitive and reversible inhibitor of inosine monophosphate dehydrogenase (IMPDH) [1, 2]. In the body, MMF is naturally hydrolyzed into mycophenolic acid (MPA), which is the active form of MMF. MMF/ MPA inhibits the de novo pathway of guanosine nucleotide synthesis without incorporation into DNA [3]. Because $\mathrm{T}$ and $\mathrm{B}$ lymphocytes depend on the de novo synthesis of purines for their proliferation, MMF/MPA has potent cytostatic effects on lymphocytes [2]. MMF has been shown to prevent the occurrence of acute rejection in rat models of kidney and heart allotransplantation [4]. MMF also decreases antibody production in mice [5]. Thus, MMF functions as a versatile immune modulator because of its broad range of actions. The biological mechanisms underlying the therapeutic effects of MMF/ MPA against rejection after organ transplantation are known to be mainly mediated by the inhibition of $\mathrm{T}$ and $\mathrm{B}$ lymphocytes, but the mechanisms by which MMF/MPA improves lupus nephritis are still largely unclear.

Dendritic cells (DCs) orchestrate both innate and adaptive immunity through cytokine-mediated and cell -cell contact mechanisms. In humans, DCs consist of two major subsets: myeloid DCs (mDCs) and plasmacytoid DCs (pDCs). They play distinct roles in innate and adaptive immunities through their capability to express specialized cytokines and molecules [6-8]. Although they account for only a small fraction of cells, pDCs represent a major source of type I interferon (IFN) in the peripheral blood and lymphoid tissues [9]. Thus, they play a central role in the innate antiviral immune response via their ability to produce vast amounts of type I IFN. This function is based on their selective expression of toll-like receptor (TLR)7 and TLR9, which respectively sense viral RNA and DNA within early endosomes [10]. Additionally, pDCs constitutively express high levels of IFN regulatory factor 7 (IRF7) [11], and type I IFN production requires IRF7 to be phosphorylated and translocated into the nucleus through rapid interaction with MyD88 and IRF7 [12-14].

Notably, a series of studies have indicated that pDCs also play a pathogenic role in the development of autoimmune diseases, such as systemic lupus erythematosus (SLE) or psoriasis, through their specialized function in type I IFN production [15-17]. SLE is a heterogeneous disease in which excessive inflammation, autoantibodies, and complement activation lead to multisystem tissue damage. The vicious pathogenic spiral responsible for the autoimmune process in SLE, likely mediated by the sustained secretion of type I IFN [15], is thought to occur as follows: pDC-derived type I IFN rapidly triggers the differentiation and activation of myeloid DCs, which in turn elicits the differentiation of autoreactive $\mathrm{CD}^{+} \mathrm{T}$ cells, $\mathrm{CD}^{+} \mathrm{T}$ cells, and B cells as antigen-presenting cells through processing self-components. These autoreactive effectors induce tissue damage (thus producing DNA/ RNA fragments) and produce auto-anti-nuclear antibody. The resultant immune complexes, such as anti-DNA antibody-self-DNA, further activate pDCs through TLRs in a sustained manner to produce type I IFN, which in turn amplifies the vicious autoimmune spiral. Consequently, pDCs and type I IFN represent specific cellular and molecular targets, respectively, in therapeutic strategies against autoimmune diseases such as SLE.

Although there is evidence indicating that MMF/MPA has immunomodulatory effects on mDCs [18-20], there are no reports showing its effects on pDCs. Therefore, the present work focused on examining the effects of MMF/MPA on the blood DC subsets, especially pDCs.

Here, we show a novel function of MMF/MPA, i.e., the ability to inhibit IFN- $\alpha$ production by human pDCs upon cellular activation with SLE serum or CpG-ODN via inhibiting the nuclear translocation of IRF7. In addition, MPA was found to suppress $\mathrm{mDC}$-derived IL12 via inhibiting STAT4 activation. Our present results suggest that MMF/MPA can interfere with the vicious pathogenic spiral of autoimmune disorders by regulating the function of DC subsets.

\section{Materials and methods Media and reagents}

RPMI-1640 supplemented with $2 \mathrm{mM}$ L-glutamine, 100 $\mathrm{U} / \mathrm{ml}$ penicillin, $100 \mathrm{ng} / \mathrm{ml}$ streptomycin, and heatinactivated 10\% FBS (Biosource International) was used for cell culturing throughout the experiments. For human cell stimulation, we used $5 \mu \mathrm{M}$ CpG-ODN 2216 (Invivogen), $1 \mu \mathrm{g} / \mathrm{ml}$ R848 (InvivoGen), and different doses of MPA (Sigma) dissolved in methanol; as a vehicle control, methanol was diluted in parallel.

\section{Cell isolation and culture}

Human mDCs and pDCs were isolated from peripheral blood mononuclear cells (PBMCs) from healthy adult donors, as described previously $[3,21]$. CD $11 \mathrm{c}^{+} /$ $\mathrm{BDCA}^{-} /$lineage $^{-} / \mathrm{CD} 4^{+}$cells (defined as $\mathrm{mDCs}$ ) and

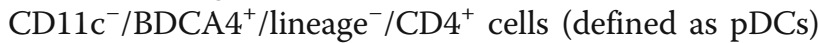
were sorted by FACS Aria ${ }^{\oplus}$ (BD Biosciences) (Suppl. Fig. 1) to reach greater than $99 \%$ purity according to restaining with anti-BDCA1 and anti-BDCA2 antibodies. The purified mDCs and pDCs were incubated for $24 \mathrm{~h}$ with R848 and CpG-ODN, respectively, in flat-bottomed 96-well plates at a concentration of $2 \times 10^{4}$ cells in a final volume of $200 \mu \mathrm{l}$ of medium per well, in the presence of MPA or the vehicle control. 


\section{Stimulation of lupus serum}

Experiments using lupus serum to stimulate pDCs were conducted as described previously [22]. Lupus sera were obtained from five active SLE patients with low complement levels prior to steroid therapy who satisfied 2019 European League Against Rheumatism (EULAR)/American College of Rheumatology (ACR) classification criteria for SLE. All patients had anti-double-stranded DNA antibody. Written informed consent was obtained from all SLE patients. In these experiments, pDCs were stimulated with $20 \%$ lupus serum in flat-bottomed 96 well plates at a concentration of $2 \times 10^{4}$ cells in $200 \mu$ of medium per well.

\section{Analyses of cells and supernatants}

Human DC subsets were stained with PE-labeled CD86 (2331) and FITC-labeled CD80 (L307.4) monoclonal antibodies (all from BD Biosciences) and then analyzed by FACScalibur ${ }^{\circ}$ (BD Biosciences). The production of cytokines in the culture supernatants after $24 \mathrm{~h}$ was determined by an enzyme-linked immunosorbent assay (ELISA). The ELISA kits for detecting human IL-12p40 and TNF were purchased from R\&D Systems. The ELISA kit for human IFN- $\alpha$ was purchased from PBL Biomedical Laboratories.

For the viability assay, cells were washed with PBS containing $2 \mathrm{mM}$ EDTA, and viable cells were counted in triplicate, using trypan-blue exclusion to identify the dead cells. Viable cells were also evaluated as the annexin V-negative fraction using an Annexin V-FITC Kit (Sigma).

For intracellular staining, pDCs were stimulated with CpG-ODN 2216 for $90 \mathrm{~min}$ or $3 \mathrm{~h}$, and the cells were immediately fixed and stained with Alexa Fluor-647 anti-pAKT (pS473; BD Biosciences) (after 90 min-culture) and Alexa Fluor-anti-human IRF-7 (G-8; sc-74472 AF488, Santa Cruz Biotechnology) (after 3-h culture) using a BD Cytofix/Cytoperm ${ }^{\text {Tx }}$ Fixation and Permeabilization Solution (BD Biosciences) according to the manufacturer's instructions. Then, the cells were analyzed by flow cytometry.

\section{Real-time PCR}

Purified DC subsets were stimulated with R848 or CpGODN in the presence of $10 \mu \mathrm{M}$ MPA for 4 or $8 \mathrm{~h}$. The cells were then collected for cDNA synthesis using the QuantiTect. Reverse Transcription (RT) kit (QIAGEN). The DNA resulting from each $\mathrm{RT}$ reaction was subjected to real-time $\mathrm{PCR}$ using the $\mathrm{SYBR}^{\circ}$ Green Master Mix (QIAGEN) for the detection of CD80, CD86, IFNA1, IL12B, TNF, IRF7, and STAT4. Primers for CD80 (QT00000497), CD86 (QT00033915), IL12B (QT00000364), IRF7 (QT00210595), and STAT4 (QT00014133) were purchased from QIAGEN. Primers for IFNA1 and TNF were purchased from Bio-Rad and Sino Biological, respectively. We selected glyceraldehyde 3-phosphate dehydrogenase (GAPDH) as the reference gene. Quantification of the mRNA transcription level was determined using the comparative cycle threshold (Ct) method. All samples were examined in duplicate, and the mean $\mathrm{Ct}$ value was calculated by Rotor Gene Q software version 2.2.3. The fold change in gene transcription was calculated as the ratio of $\Delta / \Delta C t$ in the treated samples to untreated samples.

\section{Fluorescence microscopy}

Purified pDCs were stimulated with CpG-ODN in the presence or absence of $10 \mu \mathrm{M}$ MPA for $3 \mathrm{~h}$. The cells were then seeded on glass slides by a cytospin, mounted, and fixed using a BD Transcription Factor Buffer set (Cat. No. 562725).

Samples were labeled with Alexa Fluor-anti-human IRF7 and 4',6'-diamidino-2-phenylindole (DAPI). Images were acquired using a fluorescence microscope (BZ-9000, Keyence). Cells were regarded as negative when the expression level of IRF7 in the cytoplasm was higher and distinguishable from that in the nucleus, as shown by DAPI staining. The ratio of nuclear IRF7-negative cells was calculated based on 50 cells from each donor.

\section{Statistical analysis}

Data were analyzed using paired $t$ tests. A value of $p<$ 0.05 was considered statistically significant. Data analysis was carried out using Prism (GraphPad Software).

\section{Results}

Clinical concentrations of MPA do not affect the survival of human DC subsets

Clinical pharmacokinetics show that the mean clinical trough plasma MPA concentration of lupus nephritis female patients who were administered $1500 \mathrm{mg}$ twice a day of oral MMF is $10.6 \mu \mathrm{M}(3.4 \mathrm{mg} / \mathrm{L})$ [23]. Another clinical study reported that the plasma concentration of MPA at trough in patients with severe lupus nephritis is $7.7 \mu \mathrm{M}(2.47 \mathrm{mg} / \mathrm{L})$ [24]. Therefore, we used 1-10 $\mu \mathrm{M}$ MPA (covering the clinical trough plasma concentration range for MMF/MPA) in our experiments. Concentrations of up to $10 \mu \mathrm{M}$ of MPA did not induce apoptosis of pDCs or mDCs in response to CpG-ODN or R848, respectively (Fig. 1a and b). The clinical trough concentration of MPA downregulated the R848-induced surface expression levels of CD80 and CD86 (Fig. 2a and b) and mRNA levels (Fig. 2c) in mDCs. By contrast, pDCs stimulated with CpG-ODN did not substantially upregulate either CD80 or CD86 expression (Fig. 2a), and the addition of MPA slightly but not significantly decreased these expression levels (Fig. 2b and c). 


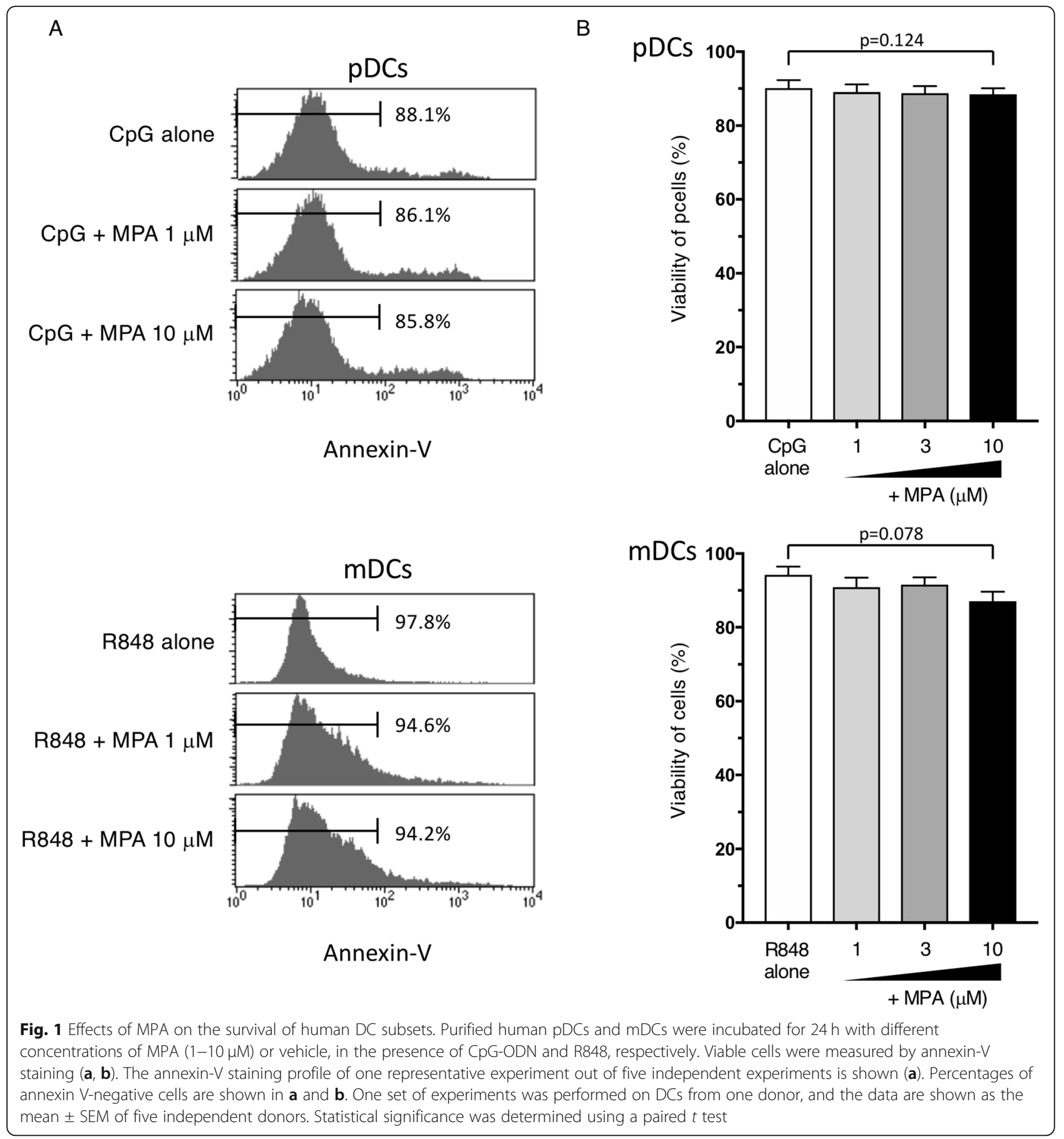

MPA inhibits IFN-a production from human pDCs

The secretion of type I IFN is a key molecular event that initiates and promotes the autoimmune process [15], and pDCs are the major source of type I IFN in PBMCs [9]. Thus, we next examined whether MPA could inhibit type I IFN production by pDCs. Notably, MPA dosedependently inhibited the production of both IFN- $\alpha$ and TNF by pDCs in response to stimulation with CpG-
ODN (Fig. 3a), and this was confirmed by transcription levels (Fig. 3b).

Immune complexes in serum consisting of autoantibodies and self-DNA are a pathogenic trigger for the induction of aberrant production of type I IFN by blood pDCs, which drives autoimmunity in SLE [15]. SLE serum also contains a nuclear protein (high-mobility group box 1 protein [HMGB1]) or an antimicrobial 


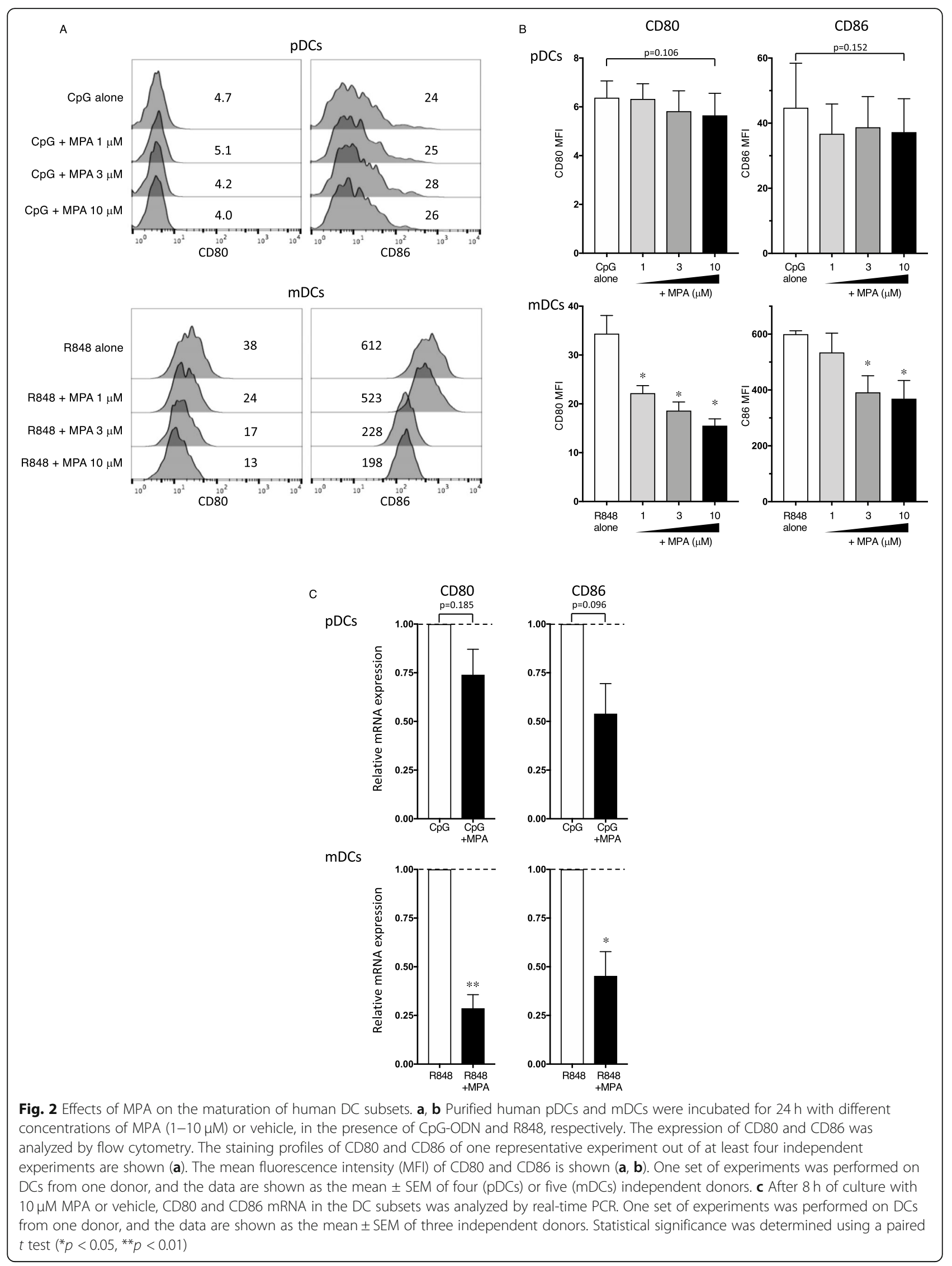




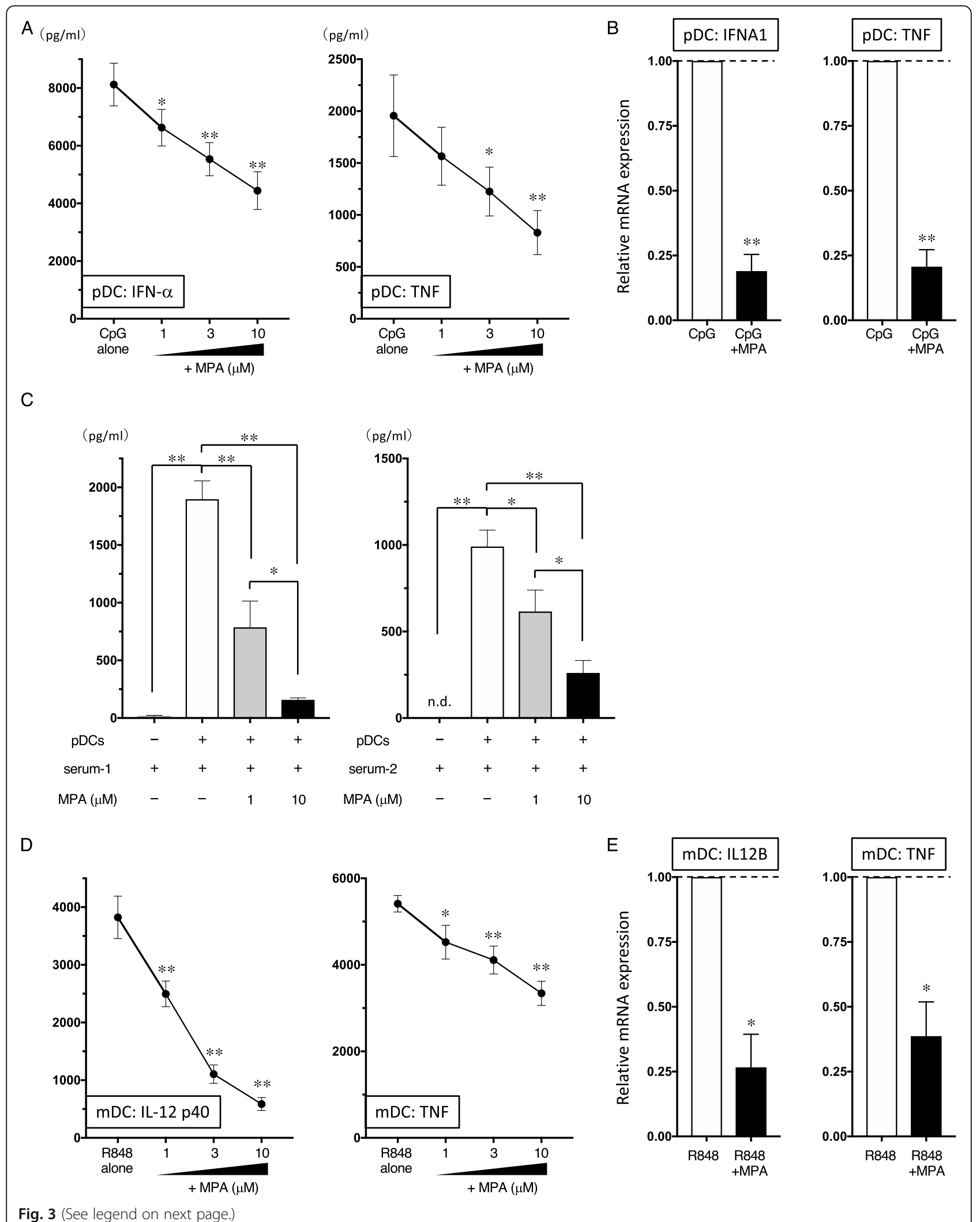


(See figure on previous page.)

Fig. 3 Effects of MPA on the ability of human DC subsets to produce cytokines. a, $\mathbf{d}$ Purified human pDCs and mDCs were incubated with different concentrations of MPA $(1-10 \mu \mathrm{M})$ or vehicle, in the presence of CpG-ODN and R848, respectively. After $24 \mathrm{~h}$, the concentrations of the indicated cytokines in the culture supernatants of pDCs (a) and $\mathrm{mDCs}(\mathbf{d})$ were measured by ELISA. One set of experiments was performed on DCs from one donor, and the data are shown as the mean \pm SEM of eight (for pDC-IFN-a), four (for pDC-TNF), five (for mDC-IL-12), or six (for mDC-TNF) independent donors. $\mathbf{b}$, e After $8 \mathrm{~h}$ of culture with $10 \mu \mathrm{M}$ MPA or vehicle, the mRNA levels of IFNA1, IL12B, and TNF were analyzed in the DC subsets by real-time PCR. One set of experiments was performed on DCs from one donor, and the data are shown as the mean \pm SEM of three independent donors. c pDCs were incubated with MPA (1 or $10 \mu \mathrm{M}$ ) or vehicle in serum-free RPMI, and serum (serum-1 or serum-2) from two SLE patients (at a final concentration of $20 \% \mathrm{vol} / \mathrm{vol}$ ) was added. After $24 \mathrm{~h}$, the concentrations of IFN-a in the culture supernatants were measured by ELISA. One set of experiments was performed on pDCs from one donor, and the data are shown as the mean \pm SEM of five independent donors. Statistical significance was determined using a paired $t$ test $\left({ }^{*} p<0.05,{ }^{* *} p<0.01\right)$. n.d., not detected

peptide LL37 released from damaged tissues or neutrophils $[15,16,25]$; these host-derived transporter agents are required for triggering the SLE pathogenic process. Thus, SLE sera possess a complete set of essential components for inducing pathogenic pDC-derived type I IFN. In previous in vitro experiments, stimulation of PBMCs with serum obtained from a patient with SLE induced IFN- $\alpha$ production [22, 26, 27]. Accordingly, we next investigated whether MPA could inhibit SLE serum-induced IFN- $\alpha$ production under conditions mimicking the pathophysiological conditions of SLE. We preliminarily tested sera from five patients with active SLE who had anti-double-stranded DNA antibody, from which we selected the two best sera for inducing IFN- $\alpha$ in healthy PBMCs (data not shown). SLE serum (either serum-1 or serum-2) induced a significant level of IFN- $\alpha$ from pDCs (Fig. 3c). Notably, concentrations of MPA of up to $10 \mu \mathrm{M}$ significantly repressed SLE serum-induced IFN- $\alpha$ production in a dose-dependent manner (Fig. 3c). These data suggest that MPA has a potential inhibitory effect on the IFN- $\alpha$ production triggered by SLE pathogenic conditions.

\section{MPA interferes with the nuclear translocation of IRF7 in pDCs}

Because the essential molecular step in type I IFN production by $\mathrm{pDCs}$ has been shown to be the nuclear translocation of constitutively expressed IRF7 [21, 28], we next examined whether MPA alters this process by evaluating both the quantitative transcriptional level of IRF-7 and the migration of IRF7 into the nucleus upon activation. First, we performed real-time PCR assays, and the results showed that the level of IRF-7 RNA in pDCs was significantly upregulated following $\mathrm{CpG}$ stimulation (Fig. 4a). Although the transcriptional upregulation of IRF-7 trended to lower following treatment with MPA, this difference did not reach statistical significance. The analysis of intracellular IRF7 expression showed similar results, with lower fluorescence intensity of IRF7 being observed in pDCs following treatment with MPA (Fig. $4 \mathrm{~b}$ and c). Analysis with immunofluorescence microscopy revealed that IRF7 was localized in the cytoplasmic area of unstimulated pDCs (Fig. 4d). After stimulation with CpG-ODN, IRF7 expression was detected in the nucleus, as shown by its colocalization with DAPI nuclear staining, indicating the nuclear translocation of IRF7. This nuclear staining of IRF7 and the colocalization of IRF7 and DAPI staining induced by CpG stimulation was prevented by the presence of $10 \mu \mathrm{M}$ MPA (Fig. 4d). Thus, MPA helped retain IRF7 in the cytoplasm, indicating its inhibitory effect on IRF7 nuclear translocation in pDCs. To quantify this finding, we counted the numbers of $\mathrm{pDCs}$ on the slide with or without nuclear IRF7 expression, as described in the "Material and methods" section and in a previous study [10]. Almost all unstimulated pDCs were found to be nuclear IRF7negative (mean \pm SEM: $95.6 \% \pm 1.2 \%$ ), and CpG stimulation significantly reduced the frequency of cells without IRF7 nuclear translocation $(12.0 \% \pm 1.4 \%)$ (Fig. 4e). Treatment with MPA significantly augmented the frequency of nuclear IRF7-negative cells following stimulation with CpG $(63.2 \% \pm 3.4 \%)$ (Fig. 4e). Thus, our result indicates that MPA acts as an inhibitor of IRF7 nuclear translocation.

To provide additional insight into possible mechanisms through which MMF is mediating the actions inhibiting the nuclear translocation of IRF7, we focused on phosphorylated AKT (pAKT) as a downstream target of phosphoinositide 3-kinase (PI3K). PI3K-pAKT pathway is reported to be closely involved in nuclear translocation of IRF7 in TLR-dependent type I IFN production of pDCs [29]. Intracellular pAKT expression in pDCs was upregulated by $\mathrm{CpG}-\mathrm{ODN}$, and this increase was repressed by the addition of MPA treatment (Fig. 5a and b).

\section{MPA inhibits Th1-related responses in $\mathrm{mDCs}$}

In the SLE pathogenic spiral, activated $\mathrm{mDCs}$ play a role in the induction of autoreactive effectors such as $\mathrm{CD} 4^{+}$ $\mathrm{T}$ cells, $\mathrm{CD}^{+} \mathrm{T}$ cells, and $\mathrm{B}$ cells. When stimulated with TLR ligands, $\mathrm{mDCs}$ are able to induce a Th1 response. To investigate the immunomodulatory actions of MPA on the function of human mDCs in Th1-related activation pathways, we analyzed the cytokine production by $\mathrm{mDC}$ that had been stimulated with R848. MPA inhibited the R848-induced production of IL-12 $\mathrm{p} 40$ and TNF 


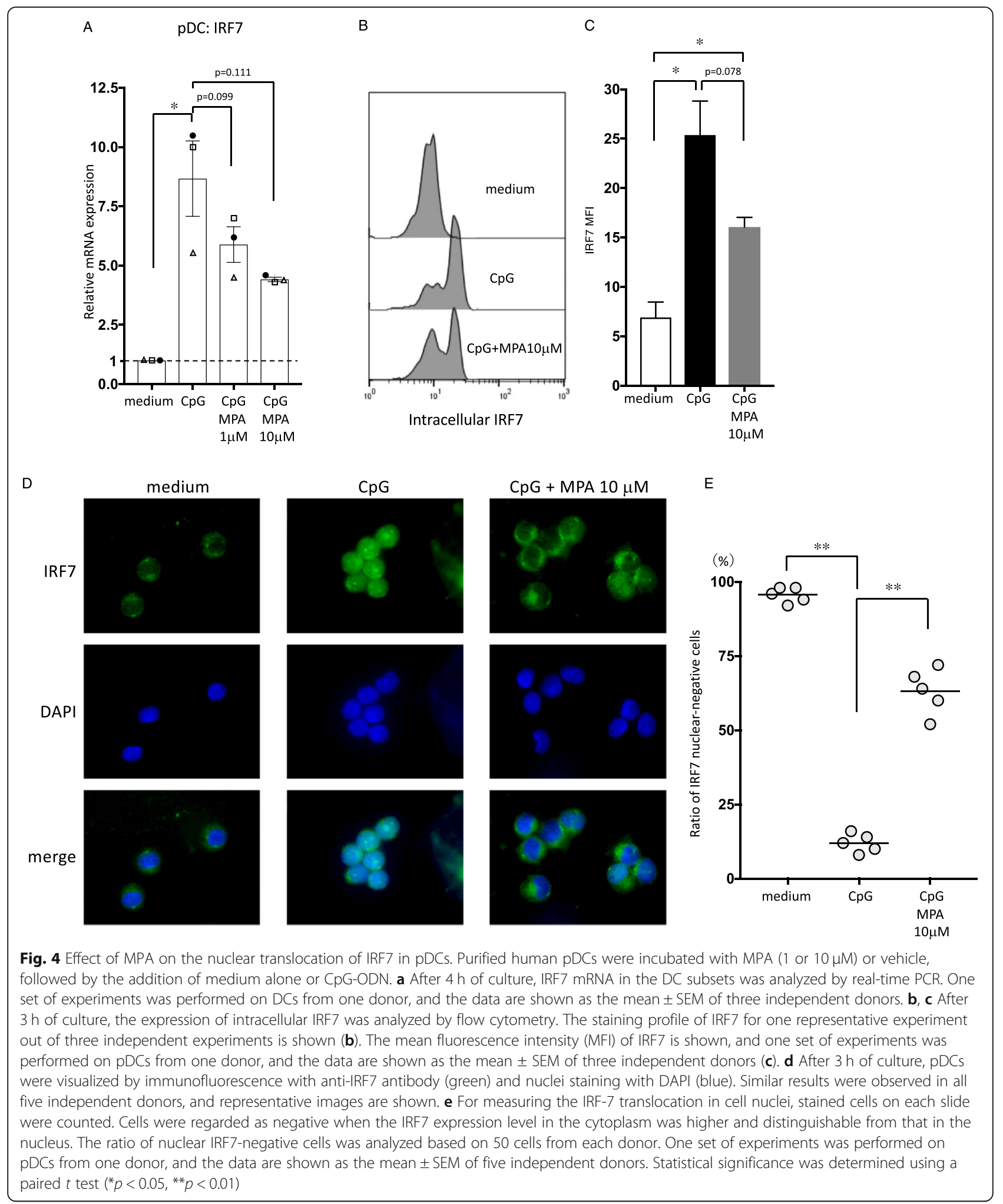


A

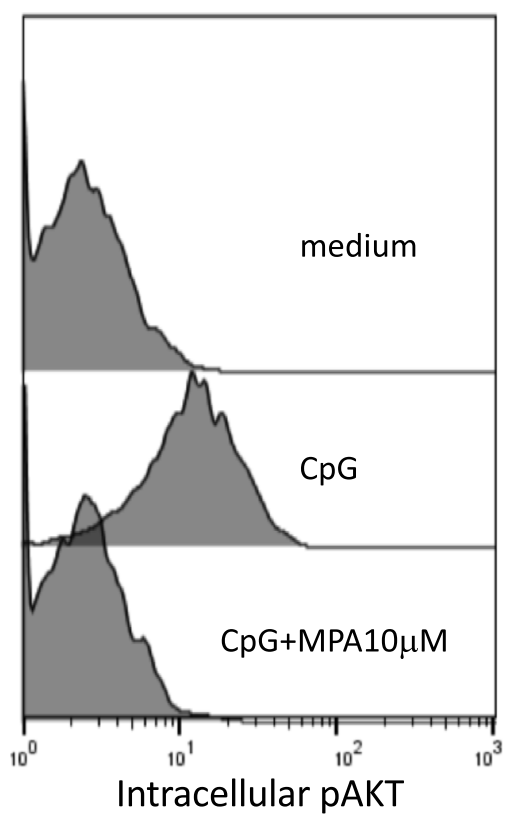

B

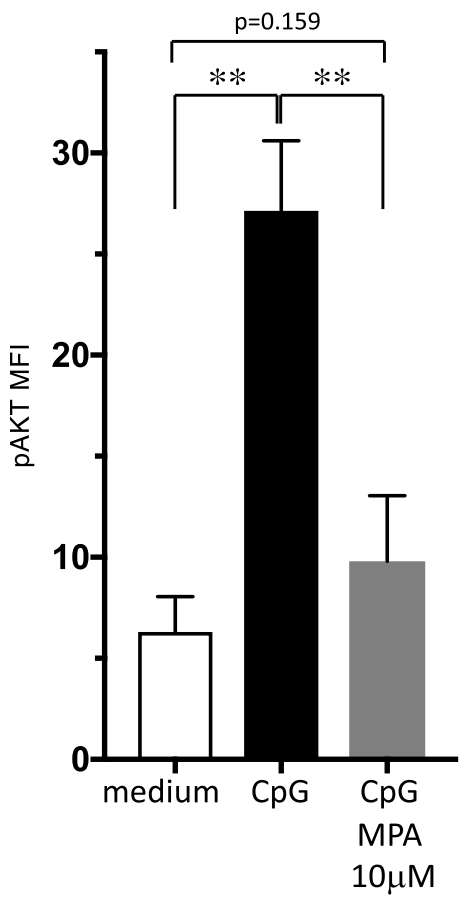

C

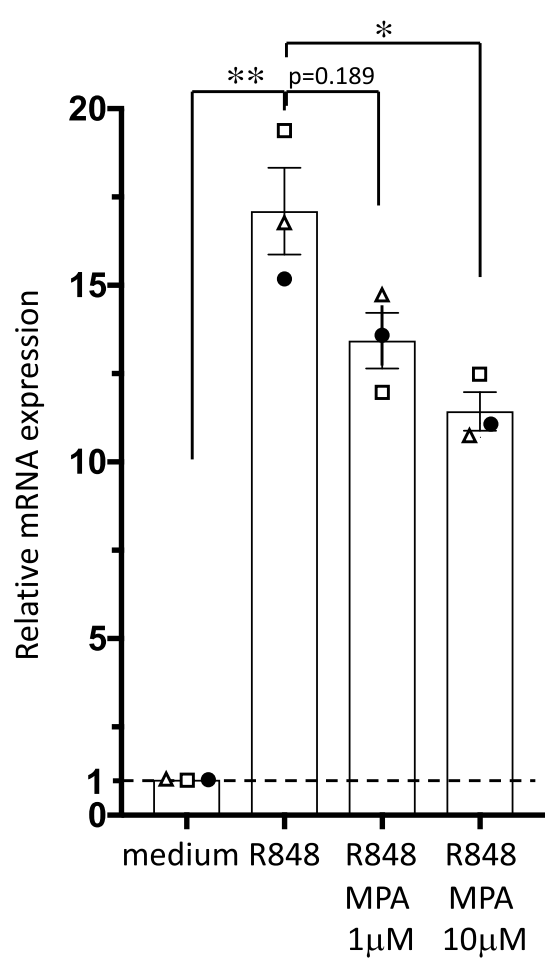

Fig. 5 Effect of MPA on intracellular molecules in DC subsets. Purified human pDCs and mDCs were incubated with MPA (1 or $10 \mu \mathrm{M})$ or vehicle, followed by the addition of medium alone, CpG-ODN, or R848. a, b After 90 min of culture, the expression of intracellular pAKT was analyzed by flow cytometry. The staining profile of PAKT for one representative experiment out of three independent experiments is shown (a). The MFI of PAKT is shown and one set of experiments was performed on pDCs from one donor, and the data are shown as the mean \pm SEM of three independent donors (b). c After $4 \mathrm{~h}$ of culture, STAT4 in the mDC subsets was analyzed by real-time PCR. One set of experiments was performed on DCs from one donor, and the data are shown as the mean \pm SEM of three independent donors. Statistical significance was determined using a paired $t$ test $\left({ }^{*} p<0.05,{ }^{* *} p<0.01\right)$

in a dose-dependent manner (Fig. 3d) and this inhibitory activity was also observed with mRNA levels (Fig. 3e). On the basis of the results of real-time PCR on mDCs, R848 significantly upregulated STAT4 mRNA, which is important for DC-mediated Th1 responses and IL-12 production [30,31]. Notably, we found that $10 \mu \mathrm{M}$ MPA significantly downregulated R848-induced STAT4 mRNA (Fig. 5c).

\section{Discussion}

In NZB mice, which develop an immune complex disease with nephritis that is similar to human SLE, MMF has a beneficial effect on both renal and overall survival [32]. MMF has been introduced as a novel immunosuppressive agent in SLE, often in patients who are intolerant or resistant to conventional immunosuppressive regimens [33, 34]. Although outlined responses are described in prior publications, the mechanism of action of MMF/MPA in the SLE pathogenic spiral remains poorly understood, except for the direct suppressive effects on $\mathrm{T}$ and $\mathrm{B}$ lymphocytes. In patients with active SLE, auto-
anti-DNA antibody, such as flares of lupus glomerulonephritis, is a common and significant marker [35-38]. Sustained activation of pDCs in the blood by immune complexes induces dysregulated type I IFN elevation, as a central molecular event, which subsequently causes $\mathrm{mDC}$ activation and the resultant induction of autoreactive lymphocytes in the vicious autoimmune spiral of SLE $[15,16]$. Here, we focused on the function of human DC subsets involved in this pathogenic spiral and unveiled the inhibitory potential of MPA against such $\mathrm{pDC}$-derived IFN- $\alpha$ production and $\mathrm{mDC}$ function. We found that MPA exerted an inhibitory action on IRF7 nuclear translocation in pDCs. It has been reported that the PI3K-AKT pathway is critically involved in the nuclear translocation of IRF7 in pDCs [29]. Our data suggest that MPA may inhibit nuclear translocation of IRF7 possibly through suppressing AKT activity in pDCs. On the basis of our results, treatment with MPA may be able to interrupt the autoimmune pathogenic spiral by limiting the disordered production of type I IFN that occurs under the pathophysiological conditions of SLE. 
Thus, the present study revealed a possible mechanism underlying the therapeutic potential of MMF/MPA for treating SLE by targeting pDCs. Notably, trough plasma concentrations of MPA did not induce cell apoptosis of either pDCs or mDCs, suggesting that MPA at clinical doses does not cause excessive immunosuppression. Hence, MMF may be useful as a long-term maintenance therapy for SLE.

Hydroxychloroquine is an effective therapeutic agent for SLE and has a mechanism similar to that of MPA; it is a strong TLR-signaling inhibitor that suppresses the production of type I IFN by pDCs [39]. MMF/MPA may exhibit cellular effects via mechanisms of action similar to those by which hydroxychloroquine treats autoimmunity [40]. Accumulating evidence has substantiated the importance of TLRs in the pathogenesis and progression of autoimmune processes in SLE and its renal components [41]. On the basis of the common properties of these two drugs, inhibition of the pDC-IFN axis may be an effective SLE treatment strategy. In fact, inhibition of TLR9 signaling with synthetic ODN can also exhibit beneficial effects on the progression of lupus nephritis in MRL ${ }^{\mathrm{lpr} / \mathrm{lpr}}$ mice [42].

The present study also revealed the ability of MPA, used at the clinical trough level, to impair the maturation and Th1inducing capacity of DCs by downregulating STAT4. In support of our findings, a recent in vitro study showed that $20 \mu \mathrm{M}$ MMF can inhibit the LPS-induced expression of MHC-class II, CD86, CD40, CD83, and NF-kB in human blood mDCs [20]. Moreover, MMF was reported to suppress the differentiation of human monocyte-derived DCs $[18,19]$. These immunosuppressive effects of MMF/MPA on mDCs may further contribute to its ability to break the pathogenic spiral of SLE.

Ideally, pDCs from the blood of SLE patients would have been used for evaluating the ability of MPA to suppress type I IFN production, triggered by pathogenic conditions. However, the blood of SLE patients has greatly reduced the numbers of circulating pDCs [16]. Furthermore, the numbers of pDCs in the bone marrow are very low in lupus-prone mice [43]. Therefore, because it was logistically and ethically difficult to perform these experiments using pDCs from SLE patients, we designed an experimental system using SLE sera.

\section{Conclusions}

To the best of our knowledge, this is the first study to investigate the effect of MMF/MPA on pDCs. This work focusing on DC function clarified one of the possible mechanisms by which MPA could be clinically effective in the treatment of SLE. Our findings suggest that MMF may be able to interrupt the vicious pathogenic spiral of autoimmune disorders, not only by inhibiting $\mathrm{T}$ and $\mathrm{B}$ lymphocytes but also by regulating the function of DC subsets.

\section{Supplementary information}

Supplementary information accompanies this paper at https://doi.org/10. 1186/s13075-020-02356-z.

Additional file 1: Supplementary Figure 1. Isolation of blood DC subsets. Blood pDCs and mDCs were detected and isolated as the $\mathrm{CD} 11 \mathrm{C}^{-} \mathrm{BDCA}-4^{+}$population and $\mathrm{CD} 11 \mathrm{C}^{+} \mathrm{BDCA}-4^{-}$population, respectively, in the fraction of DC-enriched PBMCs (lineage [CD3, CD14, CD15, CD16, CD19, and CD56]-negative and CD4-positive) after immunobead-selection (CD3- and CD14-beads negative selection and subsequent CD4-bead positive selection) from total PBMCs. A representative flow cytometry analysis performed to detect pDCs and mDCs in PBMCs from healthy donors is shown. The numbers indicate the percentages within the gated fractions.

\section{Abbreviations}

MMF: Mycophenolate mofetil; MPA: Mycophenolic acid; BDCA: Blood dendritic cell antigen; DC: Dendritic cells; IFN: Interferon; IKK: IKB kinase; IRF7: IFN regulatory factor 7; PBMC: Peripheral blood mononuclear cells; pDC: Plasmacytoid dendritic cell; SLE: Systemic lupus erythematosus;

TNF: Tumor necrosis factor; TLR: Toll-like receptor

\section{Acknowledgements}

We thank Katie Oakley, PhD, from Edanz Group (https://en-author-services. edanzgroup.com/ac) for editing a draft of this manuscript.

\section{Authors' contributions}

MS performed the experiments and wrote the paper. TI planned, designed, and wrote the paper. AS and SN contributed to the experimental planning. $\mathrm{MI}, \mathrm{TA}, \mathrm{Kl}$, and HY supported the experiments. AT, HA, TN, YS, and YO contributed to the acquisition of patient data and samples. All authors read and approved the final manuscript.

\section{Funding}

This study was supported in part by a grant (17 K09963 to T.I.) from the Grant-in-Aid for Scientific Research (C), Japan Society for the Promotion of Science.

\section{Availability of data and materials}

The data that support the findings of this study are available on request from the corresponding author (T.I.). The data are not publicly available owing to information that could compromise research participant privacy.

\section{Ethics approval and consent to participate}

All studies involving human samples were performed under institutional review board-approved protocols at Kansai Medical University (No. 2014110 and 2017208). All subjects provided written informed consent in accordance with the recommendations of the International Conference on

Harmonization Guidelines for Good Clinical Practice and the Declaration of Helsinki.

\section{Consent for publication}

Not applicable.

\section{Competing interests}

The authors declare that they have no competing interests.

Received: 7 June 2020 Accepted: 18 October 2020

Published online: 09 November 2020

References

1. Eugui EM, Almquist SJ, Muller CD, Allison AC. Lymphocyte-selective cytostatic and immunosuppressive effects of mycophenolic acid in vitro: role of deoxyguanosine nucleotide depletion. Scand J Immunol. 1991;33(2): 161-73.

2. Fulton B, Markham A. Mycophenolate mofetil. A review of its pharmacodynamic and pharmacokinetic properties and clinical efficacy in renal transplantation. Drugs. 1996;51(2):278-98. 
3. Allison AC, Eugui EM. Mechanisms of action of mycophenolate mofetil in preventing acute and chronic allograft rejection. Transplantation. 2005:80(2 Suppl):S181-90.

4. Vu MD, Qi S, Xu D, Wu J, Peng J, Daloze P, et al. Synergistic effects of mycophenolate mofetil and sirolimus in prevention of acute heart, pancreas, and kidney allograft rejection and in reversal of ongoing heart allograft rejection in the rat. Transplantation. 1998;66(12):1575-80.

5. Van Bruggen MC, Walgreen B, Rijke TP, Berden JH. Attenuation of murine lupus nephritis by mycophenolate mofetil. J Am Soc Nephrol. 1998;9(8):1407-15.

6. Ito T, Wang YH, Duramad O, Hori T, Delespesse GJ, Watanabe N, et al. TSLPactivated dendritic cells induce an inflammatory $T$ helper type 2 cell response through OX40 ligand. J Exp Med. 2005;202(9):1213-23.

7. Ito $T$, Yang $M$, Wang $Y H$, Lande R, Gregorio J, Perng OA, et al. Plasmacytoid dendritic cells prime IL-10-producing T regulatory cells by inducible costimulator ligand. J Exp Med. 2007;204(1):105-15.

8. Kadowaki N, Antonenko S, Lau JY, Liu YJ. Natural interferon alpha/betaproducing cells link innate and adaptive immunity. J Exp Med. 2000;192(2): 219-26

9. Siegal FP, Kadowaki N, Shodell M, Fitzgerald-Bocarsly PA, Shah K, Ho S, et al. The nature of the principal type 1 interferon-producing cells in human blood. Science. 1999;284(5421):1835-7.

10. Liu YJ. IPC: professional type 1 interferon-producing cells and plasmacytoid dendritic cell precursors. Annu Rev Immunol. 2005;23:275-306.

11. Honda K, Yanai H, Negishi H, Asagiri M, Sato M, Mizutani T, et al. IRF-7 is the master regulator of type-I interferon-dependent immune responses. Nature. 2005;434(7034):772-7.

12. Honda K, Yanai H, Mizutani T, Negishi H, Shimada N, Suzuki N, et al. Role of a transductional-transcriptional processor complex involving MyD88 and IRF-7 in Toll-like receptor signaling. Proc Natl Acad Sci U S A. 2004;101(43):15416-21.

13. Kawai T, Sato S, Ishii KJ, Coban C, Hemmi H, Yamamoto M, et al. Interferonalpha induction through Toll-like receptors involves a direct interaction of IRF7 with MyD88 and TRAF6. Nat Immunol. 2004;5(10):1061-8.

14. Uematsu S, Sato S, Yamamoto M, Hirotani T, Kato H, Takeshita F, et al. Interleukin-1 receptor-associated kinase-1 plays an essential role for Toll-like receptor (TLR)7- and TLR9-mediated interferon-\{alpha\} induction. J Exp Med. 2005;201(6):915-23.

15. Banchereau J, Pascual V. Type I interferon in systemic lupus erythematosus and other autoimmune diseases. Immunity. 2006;25(3):383-92.

16. Blanco P, Palucka AK, Gill M, Pascual V, Banchereau J. Induction of dendritic cell differentiation by IFN-alpha in systemic lupus erythematosus. Science. 2001;294(5546):1540-3.

17. Nestle FO, Conrad C, Tun-Kyi A, Homey B, Gombert M, Boyman O, et al. Plasmacytoid predendritic cells initiate psoriasis through interferon-alpha production. J Exp Med. 2005;202(1):135-43.

18. Colic M, Stojic-Vukanic Z, Pavlovic B, Jandric D, Stefanoska I. Mycophenolate mofetil inhibits differentiation, maturation and allostimulatory function of human monocyte-derived dendritic cells. Clin Exp Immunol. 2003;134(1):63-9.

19. Litjens NH, Rademaker M, Ravensbergen B, Thio HB, van Dissel JT, Nibbering $\mathrm{PH}$. Effects of monomethylfumarate on dendritic cell differentiation. $\mathrm{Br} J$ Dermatol. 2006;154(2):211-7.

20. Mazzola MA, Raheja R, Regev K, Beynon V, von Glehn F, Paul A, et al. Monomethyl fumarate treatment impairs maturation of human myeloid dendritic cells and their ability to activate T cells. Mult Scler. 2019;25(1):63-71.

21. Ito T, Kanzler H, Duramad O, Cao W, Liu YJ. Specialization, kinetics, and repertoire of type 1 interferon responses by human plasmacytoid predendritic cells. Blood. 2006;107(6):2423-31.

22. Miyamoto R, Ito T, Nomura S, Amakawa R, Amuro H, Katashiba $Y$, et al. Inhibitor of IkappaB kinase activity, BAY 11-7082, interferes with interferon regulatory factor 7 nuclear translocation and type I interferon production by plasmacytoid dendritic cells. Arthritis Res Ther. 2010;12(3):R87.

23. Pourafshar N, Karimi A, Wen X, Sobel E, Pourafshar S, Agrawal N, et al. The utility of trough mycophenolic acid levels for the management of lupus nephritis. Nephrol Dial Transplant. 2019;34(1):83-9.

24. Lertdumrongluk P, Somparn P, Kittanamongkolchai W, Traitanon O, Vadcharavivad S, Avihingsanon Y. Pharmacokinetics of mycophenolic acid in severe lupus nephritis. Kidney Int. 2010;78(4):389-95.

25. Ronnblom L, Pascual V. The innate immune system in SLE: type I interferons and dendritic cells. Lupus. 2008;17(5):394-9.

26. Hua J, Kirou K, Lee C, Crow MK. Functional assay of type I interferon in systemic lupus erythematosus plasma and association with anti-RNA binding protein autoantibodies. Arthritis Rheum. 2006;54(6):1906-16.
27. Lovgren T, Eloranta ML, Bave U, Alm GV, Ronnblom L. Induction of interferon-alpha production in plasmacytoid dendritic cells by immune complexes containing nucleic acid released by necrotic or late apoptotic cells and lupus lgG. Arthritis Rheum. 2004;50(6):1861-72.

28. Honda K, Ohba Y, Yanai H, Negishi H, Mizutani T, Takaoka A, et al. Spatiotemporal regulation of MyD88-IRF-7 signalling for robust type-I interferon induction. Nature. 2005;434(7036):1035-40.

29. Guiducci C, Ghirelli C, Marloie-Provost MA, Matray T, Coffman RL, Liu YJ, et al. PI3K is critical for the nuclear translocation of IRF-7 and type I IFN production by human plasmacytoid predendritic cells in response to TLR activation. J Exp Med. 2008:205(2):315-22.

30. Moser M, Murphy KM. Dendritic cell regulation of TH1-TH2 development. Nat Immunol. 2000;1(3):199-205.

31. Thieu VT, Yu Q, Chang HC, Yeh N, Nguyen ET, Sehra S, et al. Signal transducer and activator of transcription 4 is required for the transcription factor T-bet to promote T helper 1 cell-fate determination. Immunity. 2008; 29(5):679-90.

32. Corna D, Morigi M, Facchinetti D, Bertani T, Zoja C, Remuzzi G. Mycophenolate mofetil limits renal damage and prolongs life in murine lupus autoimmune disease. Kidney Int. 1997:51(5):1583-9.

33. Dooley MA, Cosio FG, Nachman PH, Falkenhain ME, Hogan SL, Falk RJ, et al. Mycophenolate mofetil therapy in lupus nephritis: clinical observations. J Am Soc Nephrol. 1999;10(4):833-9.

34. Gaubitz M, Schorat A, Schotte H, Kern P, Domschke W. Mycophenolate mofetil for the treatment of systemic lupus erythematosus: an open pilot trial. Lupus. 1999;8(9):731-6.

35. Wu JF, Yang YH, Wang LC, Lee JH, Shen EY, Chiang BL. Antinucleosome antibodies correlate with the disease severity in children with systemic lupus erythematosus. J Autoimmun. 2006;27(2):119-24.

36. Hylkema MN, van Bruggen MC, ten Hove T, de Jong J, Swaak AJ, Berden JH, et al. Histone-containing immune complexes are to a large extent responsible for anti-dsDNA reactivity in the Farr assay of active SLE patients. J Autoimmun. 2000:14(2):159-68.

37. Kippner L, Klint C, Sturfelt G, Bengtsson AA, Eriksson H, Truedsson L. Increased level of soluble HLA class I antigens in systemic lupus erythematosus: correlation with anti-DNA antibodies and leukopenia. J Autoimmun. 2001:16(4):471-8.

38. Okamura M, Kanayama Y, Amastu K, Negoro N, Kohda S, Takeda T, et al. Significance of enzyme linked immunosorbent assay (ELISA) for antibodies to double stranded and single stranded DNA in patients with lupus nephritis: correlation with severity of renal histology. Ann Rheum Dis. 1993; 52(1):14-20.

39. Sacre K, Criswell LA, McCune JM. Hydroxychloroquine is associated with impaired interferon-alpha and tumor necrosis factor-alpha production by plasmacytoid dendritic cells in systemic lupus erythematosus. Arthritis Res Ther. 2012;14(3):R155.

40. Schrezenmeier E, Dörner T. Mechanisms of action of hydroxychloroquine and chloroquine: implications for rheumatology. Nat Rev Rheumatol. 2020 16(3):155-66.

41. Conti F, Spinelli FR, Alessandri C, Valesini G. Toll-like receptors and lupus nephritis. Clin Rev Allergy Immunol. 2011;40(3):192-8.

42. Patole PS, Zecher D, Pawar RD, Gröne HJ, Schlöndorff D, Anders HJ. G-rich DNA suppresses systemic lupus. J Am Soc Nephrol. 2005;16(11):3273-80.

43. Scott JL, Wirth JR, EuDaly JG, Gilkeson GS, Cunningham MA. Plasmacytoid dendritic cell distribution and maturation are altered in lupus prone mice prior to the onset of clinical disease. Clin Immunol. 2017;175:109-14.

\section{Publisher's Note}

Springer Nature remains neutral with regard to jurisdictional claims in published maps and institutional affiliations. 